\title{
Morphological Evaluation and Grading of Human Embryo Quality from Day - 1 to Day - 3 Embryos for Optimum Conceiving Rate
}

\author{
Ram Dayal ${ }^{1}$, Sumer Singh ${ }^{2}$, Prabhat Kumar ${ }^{3}$, Kamla Singh ${ }^{4}$ Pawan Kumar Tripathi ${ }^{5}$, \\ Rajni Gupta ${ }^{6}$, Sumit Singhal ${ }^{7}$
}

${ }^{1}$ Research Scholar in Biotechnology, Singhania University, Pacheri Bari, (Jhunjhunu), Rajasthan, India

${ }^{2}$ Associate Prof. \& Head School of Life Sciences, Singhania University, Rajasthan, India

${ }^{3}$ Associate Professor, School of Life Sciences, Singhania University, Rajasthan, India

${ }^{4} \mathrm{CMD} \&$ Infertility Consultant, IRCC Hospital, Panchkula, Haryana, India

${ }^{5}$ Associate Prof. \& Head Research Dept, Singhania University, Rajasthan, India

6IVF Specialist \& Head, Nitin IVF Centre, Nitin Hospital, Patiala, India

${ }^{7}$ Assistant Prof., Vet. OBG, GADVASU, Ludhiana, India

Corresponding Author Email id : ramdayal2509@gmail.com ${ }^{1}$

\section{Article Info}

Volume 7, Issue 5

Page Number: 225-236

Publication Issue :

September-October-2020

\section{Article History}

Accepted : 01 Oct 2020

Published : 05 Oct 2020

\section{ABSTRACT}

A grading system of human embryo is very important for embryo selection \& predicting blastocyst formation from day - 1, day 2 , \& day -3 , were sequentially explained. We were designed a methods for grading of embryos from day - 1 (zygote as a pronuclear evaluation, PN), day - 2 (cleavage stage) and day - 3 (evaluation of developmental stage according to cell size and fragmentation) embryo according to cell size and degree of fragmentation and early compaction. In the first grading system pronuclear study and polyspermy, Second grading system is based on the blastomere / cell number and the observation of fragmentation pattern and selection for embryo transfer, embryos vitrification and pregnancy outcome. Assessment of embryo quality in order to select the embryos that have higher chance to give pregnancy, it is critical goal in IVF cycle or assisted reproductive technologies. ET current trend in human infertility treatment with IVF / ICSI embryo transfer (IVF / ICSI ET) is to increase the chance of higher pregnancy and reduce the multiple pregnancies after multiple embryo transfer according to patient age and endometrium thickness as well as own ART Laboratory protocol. Morphological evaluation \& grading of human embryo as a reliable and noinvasive method that provides valuable information \& prediction of IVF/ICSI embryos which has developmental potential to reach till early compaction or blastocyst. This research paper describes the current status of morphological embryo evaluation from zygote to eight cell blastomeres or early compaction on late day 3 . 
We found higher embryo development potential and early compaction during in-vitro embryo culture conditions and higher implantation rate in Grade A embryos in comparison transferred of Grade B embryos. Overall embryo development in-vitro and conceiving rate was seen $\mathbf{4 8 . 0 6 \%}$ after embryo transfer of both grades A and Grade B embryos in 233 patients in different age group with different endometrium thickness and multiple embryos transfer in one uterus depend on patient previous history. 935 Embryos was selected from 2702 developing embryos for embryo transfer (ET) was performed in 233 patients. After this study we found implantation rate (IR) was $48.06 \%$ based on embryo quality, morphology and grade.

Keywords : IVF / ICSI, Fertilization, Zygote, Cleavage Stage, Day - 2, \& day - 3 embryos grade, Embryo Morphology, Embryo Transfer (ET) \& Vitrification

\section{INTRODUCTION}

Successful outcome after IVF/ICSI procedures depend on many factors, one most critical factor being embryo quality and grade. Embryo grading system has many potential benefits. Such as (1) - proper grading and scoring of pronuclei (2PN, 3PN), (2) - proper embryo selection of transfer, (3) -Standardizing embryos grading system by embryologist, (4) Reducing high order of multiple birth by adjustment of transfer number of embryos according to embryo grade or quality, (5) - Permitting comparison of embryo quality between patient cycle and (6) evaluation of culture regimens. Application of proper embryo scoring system has many potential benefits such as: 1 . accurate selection of embryos prior to ET, 2 . Reduction of the risk of twin / multiple pregnancies, 3. Assessment of embryo development and culture.

Accurate selection of embryos for transfer and prediction of implantation is the most important goal in assisted reproduction cycle. Generally, quality and developmental rate of human embryos that are produced in-vitro may vary broadly. These differences may indicate the inherent developmental potential in the gamete as well as IVF / ICSI techniques and culture conditions. The success rate of
IVF is depends on many factors such a quality of gametes, quality of embryos, patients conditions, patient age, endometrium thickness, embryo selection techniques and laboratory condition. A lower number of embryo transfers can decrease the chance of pregnancy. However, if the goal is to increase pregnancy rate with restricting possibility of multiple pregnancies, more sensitive and non invasive methods are required for embryo grading and selection prior embryo transfer.

Current embryo grading systems differ with regards to selection of embryo grade or stage and criteria for assessment of embryo quality. There are several stages for evaluation of pre-implantation embryo quality. In this study we have received some precise protocols in each stage.

\section{Overview of the research}

We have done a research on the human developing embryos especially ongoing zygote to blastocyst as in the manner of comparison which embryo has ability to develop in blastocyst (more chance for conception) in vitro. Those embryos; I had took through in vitro fertilization (IVF) in the field of assisted reproductive technology (ART) after patient couples consent for 
research purpose. Now a day's many couples does not have natural conception because male and female infertility both is included. Those patients can achieve a conception through ART. But in these technique we required for best selection of embryos from day 1 (2PN, zygote), day 2 (Cleavage), day 3 (developmental stage) based on cell size and degree of fragmentation or other deformity in the embryos. Best selection of embryo for embryo transfer (ET) is more chance to increase conception; embryo quality is the best predictive factor for pregnancy in IVF / ICSI cycles.

\section{Research design, material \& methods}

1. IVF lab with fully AC and power backup as well as sterility should be maintained.

2. Prepared patient is available for ovum pickup (OPU) self or donor.

3. Hera cell $150 \mathrm{i} \mathrm{CO}_{2}$ incubator or any other $\mathrm{CO}_{2}$ incubator.

4. IVF Work station, heating stage with steriozoom microscope.

5. $\mathrm{CO}_{2}$ Analyzer K-System or Geotech.

6. Inverted Microscope with micromanipulator

7. Binocular Compound / phase contrast Microscope.

8. Culture media From Irvine Scientific: -

a) Modified Human Tubal Fluid Media (mHTF)

b) Continuous Single Culture Media (CSC) NGS

c) Oil for Embryo Culture

d) Polyvinyl propylidone (PVP) $7 \%$ or $10 \%$

e) Hyaluronidase

9. Sperm wash media (Puresperm Grad II from Cryolab International)

10. Culture Dish like as (FALCON) large round dish (100*20), FALCON Centre well dish (3037), NUNC Four well dish (4444), FALCON small dish (3001 and 3002) etc.

11. FALCON Transfer pipette (7575)
12. Denuding pipette $-140 \mu \mathrm{l}, 175 \mu \mathrm{l}$ and $300 \mu \mathrm{l}$ from COOK.

13. Stripper Handle from $\mathrm{COOK}$

14. 0 to $20 \mu \mathrm{l}, 5$ to $50 \mu \mathrm{l}, 10$ to $200 \mu \mathrm{l}, 100 \mu \mathrm{l}$ to 1000 $\mu \mathrm{l}$ pipette.

15. One freeze box for liquid nitrogen (LN2)

16. Ovum Pickup (OPU) Needle from cook.

17. Embryo Transfer Catheter (Sydney IVF ET Catheter, cook)

18. $1 \mathrm{ml}$ B.D. syringe or $1 \mathrm{ml}$ insulin syringe.

19. $2 \mathrm{ml}$ B.D. syringe rubber free.

20. $5 \mathrm{ml}$ B.D. syringe rubber free.

21. Gilson pipette tips individual sterile packing.

22. Semen collection container wide mouth with gama sterile.

23. Round bottom plunger.

24. Makler counting chamber (Sefi Medical Instrument, Israel)

25. Test tube warmer (Keten / Mobile nest from shivani scientific)

26. Digital oocyte aspiration suction pump from Cook.

27. Sterile powder free gloves.

28. Sterile OT Dress.

29. Sterile cap

30. Sterile mask

31. Sterile OT Sleeper

32. Tissue paper

\section{Ovarian Stimulation \& Oocyte Retrieval (OCR)}

Down regulation for ovarian stimulation were achieved by using either a gonadotropin-releasing hormone $(\mathrm{GnRH})$ agonist or antagonist protocol, starting on either menstrual cycle of Day 1 or Day 21 by Infertility Specialists Dr. Kamla Singh (IRCC Hospital \& IVF Centre) \& Dr. Rajni Gupta (Nitin Hospital \& IVF Centre). Following down regulation, ovarian stimulation were induced by using either recombinant FSH or urine derived menopur. The starting dose will be depending on woman's age or 
previous response to ovarian stimulation. The ovarian response was monitored by means of serum estradiol (E2) assays and vaginal ultrasonographic scans of follicles. Recombinant FSH or menopur were administered until the leading follicles were reaching at least $17 \mathrm{~mm}$. For maturation of the oocyte triggering by subcutaneous (SC) injection of hCG. Oocyte were retrieved by transvaginal needle aspiration (opu needle Ref \# K-OPS-1735-A-L COOK Australia) under ultrasonic guidance $36-38 \mathrm{hrs}$ post hCG. The cumulus oocyte complexes shall randomly allocate to continuous single culture medium (CSC) (Irvine Scientific).

Before OCR or Ovum pickup (OPU) all culture medium and consumable should be required overnight equilibration like as:-

\section{Modified Human Tubal Fluid Media (m HTF)} Without $\mathrm{CO}_{2}$ at $37^{\circ} \mathrm{C}$

2. Continuous Single Culture (CSC) Media with $6 \%$ $\mathrm{CO}_{2}$ at $37^{\circ} \mathrm{C}$

3. Oil for Embryo Culture with $6 \% \mathrm{CO}_{2}$ at $37^{\circ} \mathrm{C}$

4. Mobile nest or test tube warmer should be on 4 hrs prior use.

5. Respective dishes should be kept in the incubator at $37^{\circ} \mathrm{C}$ over night.

At the expected time of egg rupture after hCG (35-38 hrs) were receive the patient in IVF OT in a precise manner and start the ovum pickup (OPU) under general anesthesia by infertility surgeon and screened the oocyte under the steriozoom microscope by the Embryologist / Research Scholar / Scientist.

After screening we can do the scoring of oocyte on the basis of expansion of cumulus:
Equilibration of Oocyte : After OPU and oocyte screening all oocyte wash $2-3$ times in the HTF medium in separate dish and transfer in the bicarbonate buffer medium or IVF medium for equilibration at least $2-4$ hours. Equilibration helps in the oocyte maturation \& reducing the manipulation stress of the oocyte. After equilibration, oocyte with cumulus are ready for doing conventional IVF or ready for ICSI (after denudation) procedure. After IVF / ICSI must wash the oocyte and transfer in new bicarbonate buffer medium for culture till day 3 or till blastocyst or till ET / vitrification.

During oocyte equilibration, we prepare semen sample for conventional in-vitro fertilization (IVF) or ICSI as required for the patient. According to WHO manual - 2010 semen parameters; volume $-1.5 \mathrm{ml}$ or more, $\mathrm{pH}-7.2$ or more, concentration 15 million or more and sperm motility both forward (type a), slow progressive $(\mathrm{b}) \geq 32 \%$ and leukocyte $\leq 1$ million. 100000 sperms per oocyte are required for fertilization in IVF and one sperm is required for fertilization in ICSI.

\begin{tabular}{|l|l|l|l|}
\hline \multirow{2}{*}{} & \multicolumn{3}{|c|}{ GRADE } \\
\cline { 2 - 4 } & \multicolumn{1}{|c|}{$\mathbf{2}$} & \multicolumn{1}{|c|}{$\mathbf{3}$} \\
\hline \multirow{2}{*}{ DAY 0 } & Fully & Partially & Not \\
expanded & expanded & expanded \\
cumulus & cumulus & cumulus \\
and golden & and & and dark \\
brown & coronal & ooplasm. \\
& colour. & cells & Likely to be \\
Likely to & & an \\
be a mature & & immature \\
egg & & egg \\
\hline
\end{tabular}




\section{Semen Parameters, Preparation and Fertilization:}

All semen samples were analyzed after completing the liquefaction and prepare sample for IVF or ICSI procedures with double density gradient (DDG) or single density gradient (SDG) depending on semen parameters. Preparation was being carried out in Pure Sperm Grade II media (Cryolab international). Briefly: liquefied semen were gently overlaid the double density or single density gradient for 12 minutes at $1500 \mathrm{RPM}$. The supernatant was aspirated and pellet was transferred into a new centrifuge tube with $4 \mathrm{ml}$ washing media (HTF) and re-centrifuge 5 minutes at 1000 - 1500 RPM. Discard the supernatant by leaving pellet only and overlay with IVF culture medium and keep it in 6\% $\mathrm{CO}_{2}$ incubator at least 2030 minutes for good swim up in slanting position. Sperm concentration was determine by pipetting an aliquot of $5 \mu \mathrm{l}$ swim up sample onto a Makler Counting Chamber (Sefi Medical Instruments, Israel), followed by counting with $20 \mathrm{x}$ objective under a phase contrast microscope preferably.

IVF : Conventional IVF were performed in normal semen parameters samples. IVF can be doing in micro drop culture $(21 \mu \mathrm{l}$ to $50 \mu \mathrm{l})$ or in a well culture (500 $\mu \mathrm{l})$ continuous single culture medium (Irvine Scientific) under oil for incubation and fertilization. It can be a short period of incubation or it can be a long period of incubation, it depends on embryologist experience. In short protocol of IVF oocyte can be denuded after 3 to 5 hours of insemination, in this protocol sperm count and motility needs very good and needs to add swim up sample 100000 sperms per oocyte. In long protocol of conventional IVF we need good quality normal motile sperm used for insemination around 60000 to 100000 sperms per oocyte for long incubation overnight or not more than 18 hours. In such a way after denudation if some oocyte not shown $2 \mathrm{~PB}$ or $\mathrm{PN}$ then can be do the rescue ICSI (optional). In micro drops (30 to $50 \mu \mathrm{l}$ ) is sufficient for 1 to 3 or 4 oocytes and in well culture $500 \mu \mathrm{l}$ is enough for 5 to 10 oocytes. It is varying lab to lab and embryologist working skills.
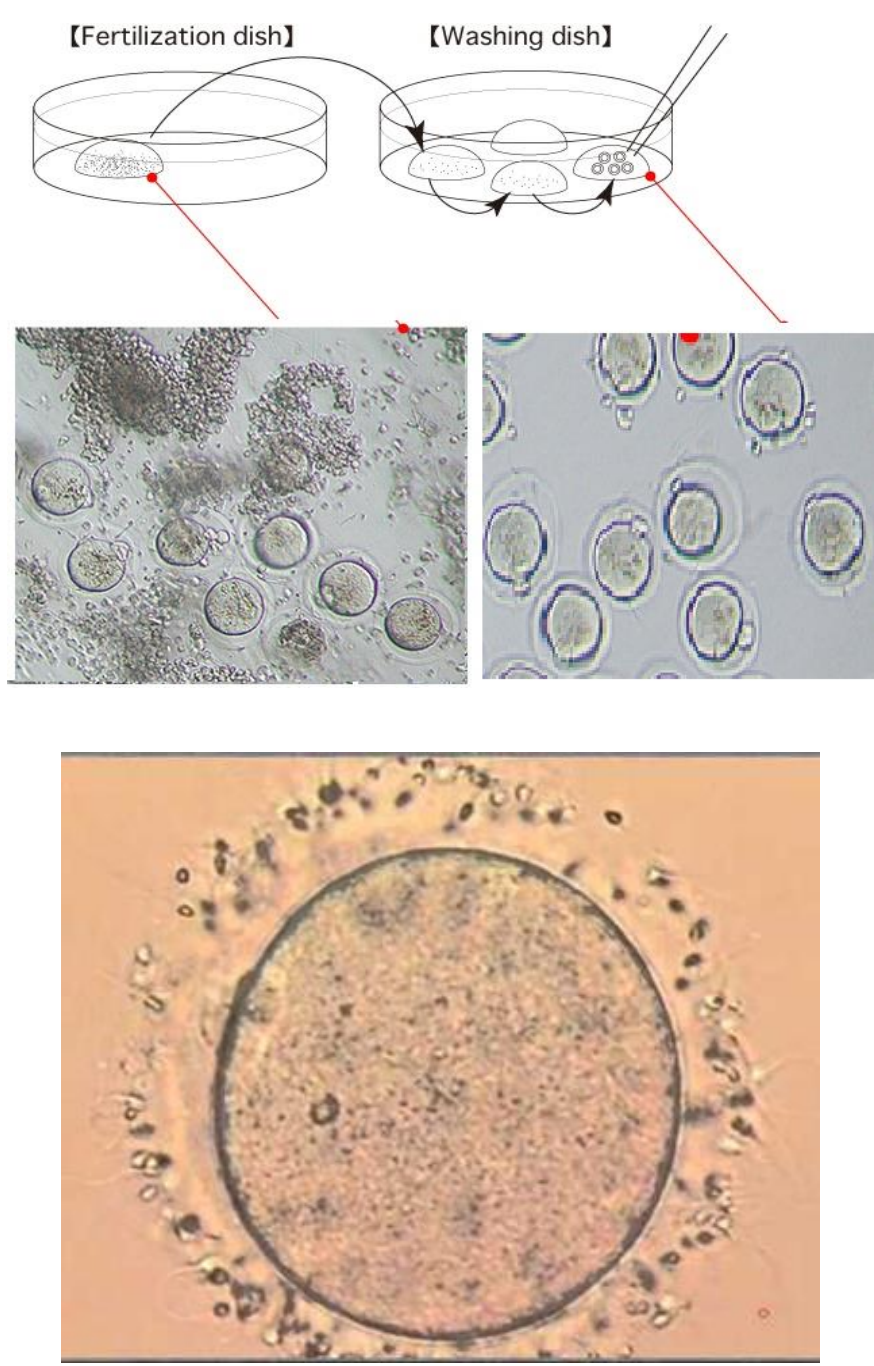

Fig 1. IVF dish, Denudation and washing dish

IVF dish looking like above figure after short incubation 3-5 hours of sperm insemination and if almost all cumulus will removed itself it is the good sign of fertilization has been done, if cumulus is associated with the zona like as above 1-2 oocytes are more cumulus is attached it means it is not fertilized \& its needs to more longer incubation. Otherwise fertilization should be fail. In second figure or dish oocyte looks very good after denudation and also showing two polar bodies after 3-5 hours of 
insemination. One last oocyte showed two polar bodies after 3 hours of insemination.

Denudation: We were denuding the oocyte between 3-5 hours after insemination or overnight equilibration, when cumulus is separated or leaves the cumulus from the oocyte. We were used different size of denuding pipette; on day 0,135 to 150 micrometer diameter, on day 2 or 3, 170 micrometer and day 4 or 5, 270 to 300 micrometer. In this research I was denuded after 5 hours of insemination (IVF); short period of incubation and I was seen $99 \%$ fertilization.

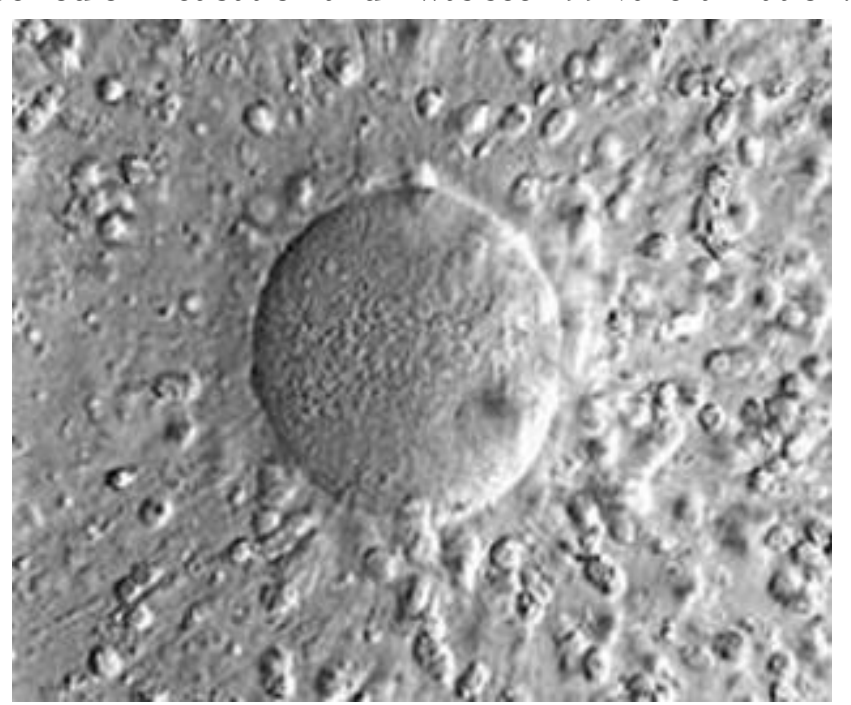

Fig 2 : Oocyte with Cumulus for IVF

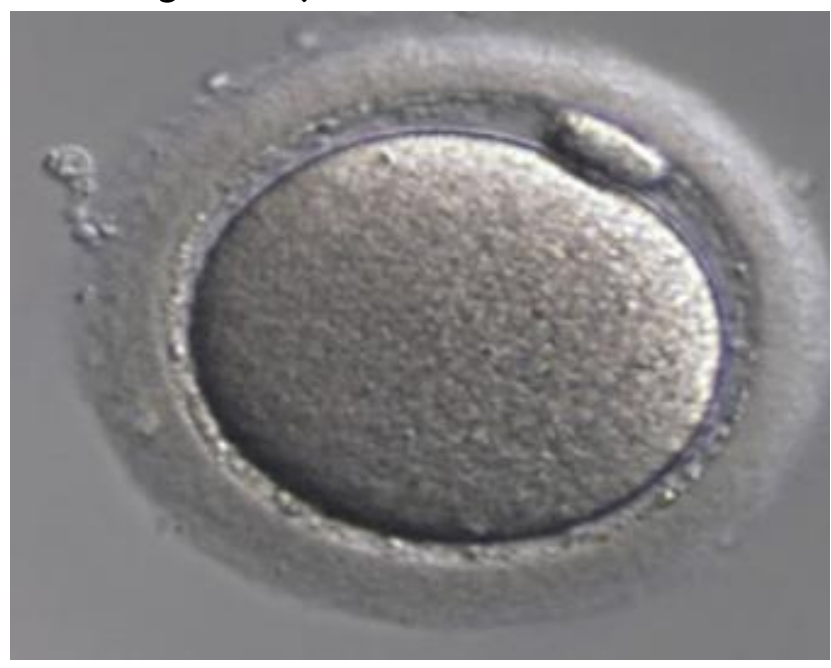

Fig 3 : Denuded Oocyte with MII stage for ICSI

\section{In vitro fertilization with Intracytoplasmic sperm injection (ICSI):}

I was performed Intracytoplasmic Sperm Injection (ICSI) only those patients who had poor sperm quality, poor sperm morphology, oligoasthenozoospermia, TESA/MESA/PESA sample, failed IVF, poor sperm survival or higher DFI. Prior ICSI procedure denudation of oocyte must be required and only MII oocyte was used for ICSI.

Denudation of oocyte for ICSI: It was done in hayaluronidase enzyme (by using 200 micro liter pipette tips initially then use $140 \mu \mathrm{l}$ denuding pipette within 40 seconds in concentrated hyaluronidase and 80 seconds in 1:1 diluted hyaluronidase) and wash the oocyte in HTF medium minimum three times after that again wash the denuded oocyte in continuous single culture medium minimum two or three times and then transfer the oocyte in micro culture dish and transfer the dish in $6 \% \mathrm{CO}_{2}$ incubator at $37^{\circ} \mathrm{C}$ for equilibration and reducing stress. Before ICSI I was note down the quality of oocyte as well as how many oocytes at matured (MII) and immature (MI) stage. During oocyte equilibration I was align the micromanipulator machine and remove the air from oil injector and fill the oil in the injector if needed. Set the injection needle as well as holding pipette by using lower objective lens (4X or $10 \mathrm{X}$ ). After fitting the needle need to leave oil injector slightly in positive pressure for short time. In mean time I was make an ICSI dish and add less than $1 \mu$ sperms in one line of polyvinylpyrrolidone (PVP). This semen sample was already prepared and done the swim up after OPU, during oocyte equilibration. After 16 to 18 hours of incubation we were seen sign of $2 \mathrm{PN}$ as a good fertilization result.

- 2 Pronuclei (2PN)

- 2 Polar Bodies (2 PBs)

- 1 day after egg retrieval 


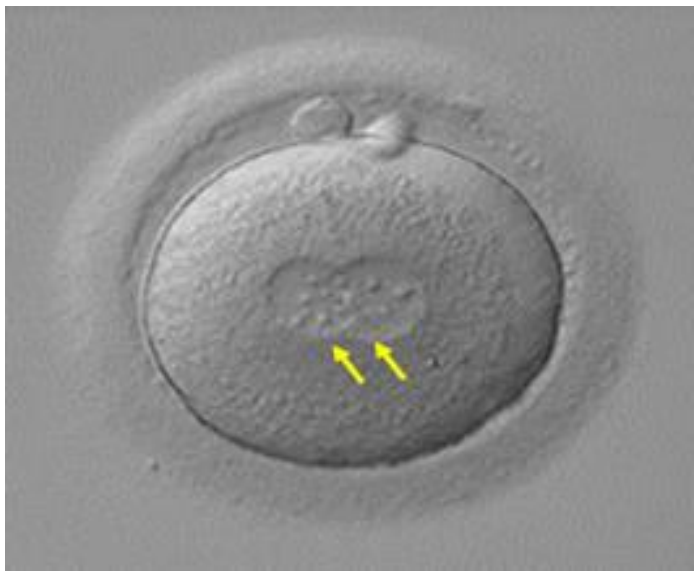

Fig 4 : Zygote

\section{Embryo culture \& morphological evaluation or grading on specific time :}

During the study period the culture conditions kept identical as regard temperature, $\mathrm{pH}$ and percent of $\mathrm{CO}_{2}$. To ensure optimal fertilization and culture conditions in the incubator, daily manual temperature readings were recorded. The $\mathrm{pH}$ of the media were check once in a week or before use of new batch culture media and the $\mathrm{CO}_{2}$ level were adjusted for optimal $\mathrm{pH}$ range of 7.2 to 7.4. We used these criteria, $\mathrm{pH}$ for all patient embryo developments as recommended by the manufacturer.

Following fertilization post IVF or ICSI, zygote were evaluated and cultured in groups of $5-10$ under oil in $500 \mu \mathrm{l}$ of $\mathrm{CSC}$ at $37^{\circ} \mathrm{C}$ in a $6 \% \mathrm{CO} 2$ incubator or in micro culture of $50 \mu \mathrm{l}$ of CSC medium under oil. In micro drop culture we were cultured 1 to 3 zygote or embryos.

\section{Day - 1 embryo (2PN or zygote) study:}

When we were reached in IVF lab morning on day $1^{\text {st }}$ of embryo culture, first of all we were checked the percentage (\%) of $\mathrm{CO}_{2}$ inside the incubator by using $\mathrm{CO}_{2}$ analyzer and also record the temperature on daily record book. We were recorded humidity and IVF lab environment temperature and switch off the coda air filter and AC before take out the culture dish from the incubator for checking the embryo status and developments.

On the morning of the day 1 on specific time was checked the pro nuclei (PN) \& scoring on the basis of PN size, Nuclear Alignment, Nucleolar alignment, Distribution and position of nuclei.

The most important predicting factor of normal embryo development in the zygote stage is the visualization of two pronuclei (2 PNs), one from sperm cell and one from oocyte. It should occur between 16 to 19 hour post IVF / ICSI.

The morphological feature of each zygote was evaluated under inverted microscope. The zygotes were classified as flows:

\section{PN size}

2. Nuclear Alignment

3. Nucleolar alignment

4. Distribution nucleoli

5. Position of nuclei.

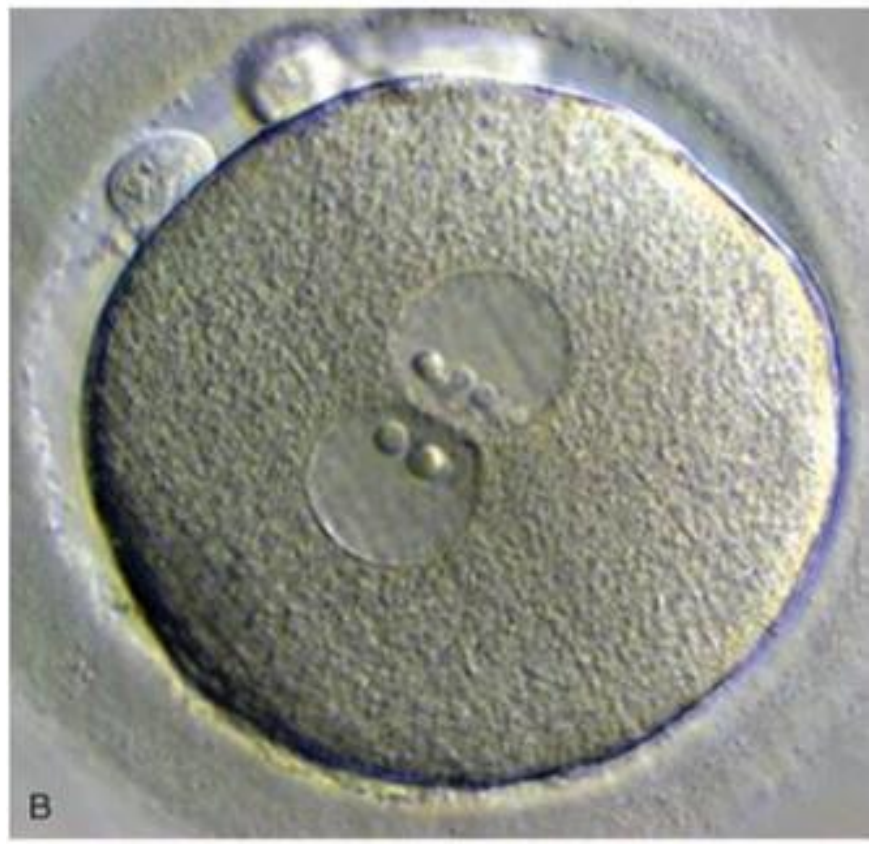

Fig 5 : Fertilized Egg (Zygote), 2 Polar Bodies, 2 Pro Nuclei (2PN), Day 1 Embryo 
Table 1 : Evaluation of pronuclear (PN) stage after 16$20 \mathrm{hr}$ (day-1) of IVF

\begin{tabular}{|c|c|}
\hline Position of PN & PN score (Z1 to Z4) \\
\hline $\begin{array}{c}\text { NPB's in Center (PN) \& } \\
\text { equal no. }\end{array}$ & $\mathrm{Z} 1$ \\
\hline $\begin{array}{c}\text { NPB's in Non Center (PN) } \\
\text { \& unequal }\end{array}$ & $\mathrm{Z} 2$ \\
\hline $\begin{array}{c}\text { NPB's in Non Center (PN) } \\
\text { \& Separate }\end{array}$ & $\mathrm{Z} 3$ \\
\hline $\begin{array}{c}\text { NPB's in Non Center (PN) } \\
\text { \& different in size }\end{array}$ & \\
\hline
\end{tabular}

\section{Day - 2, Embryo (2 or 4 cells) study}

When I was reached in IVF lab in the morning on day $2^{\text {nd }}$ of embryo culture, first of all I was checked the percentage (\%) of $\mathrm{CO}_{2}$ level inside the incubator by using $\mathrm{CO}_{2}$ analyzer and also record the temperature on daily record book. I was recorded humidity and IVF lab environment temperature and switch off the coda air filter and AC before take out the culture dish from the incubator for checking the embryo status and developments.

On specific time in the morning of day 2, I was check the cleavage stage on the basis of cell size, no. of cells/blastomeres, periviteline space and degree of fragmentation.

25 to 48 hours after the fertilization embryos reach 2cell to 4 cell stage of their development. There are very few studies focusing on this stage and on its impact on the positive implantation or reaching blastocyst in vitro. Most of them presented the conclusions that assessment of 4-cell embryo stage together with the subsequent stages of embryo development can present additive positive value in embryo reproductive potential and implantation rate. The perfect 4-cell embryo should have equal size of regular symmetrical blastomers with minimal fragmentation. The morphological feature of each embryo cleavage was evaluated under inverted microscope by using 20X / $40 \mathrm{X}$ objectives. The embryos were classified as flows:

1. A normal zygote

2. Normal embryos with cell size and cleavage rate

3. Embryos with no fragmentation.

4. Embryos with abnormal development

Table 2 : Evaluation of cleavage stage on day2 after IVF

\begin{tabular}{|c|c|}
\hline Cell stage & Grade \\
\hline 2 -cell & $\mathrm{AB}$ \\
\hline 4 -cell & $\mathrm{A}$ \\
\hline
\end{tabular}

Day - 3, embryo (Cleavage or development) study:

In the morning of day $3^{\text {rd }}$ on specific time was checked the cleavage stage; 8-celled stage of embryo development should be confirmed. The assessment of Day-3 embryos includes the number of cells, the symmetry of blastomers, the degree of fragmentation and eventually the early embryo compaction. Many studies confirmed that relationship between the numbers of blastomers observed on third day after fertilization they are tightly attached to each other cells and they were showed development of early compaction, blastocyst rate formation in-vitro as well as were seen implantation rate following embryo transfer. Some authors suggest that embryos having exactly 8 blastomers on the third day, in those embryos present highest reproductive potential. Those embryos have the highest chance of blastocyst formation in-vitro and higher chance of implantation following embryo transfer.

Selection of top quality embryos on day-3 is characterized as: 
- 8-cell embryo

- Equal blastomeres

- No / minor fragmentation

- No multi-nucleation

Table 3 : Evaluation of cleavage stage on day3 after IVF

\begin{tabular}{|c|c|}
\hline Cell stage & Grade \\
\hline 4 -cell & C \\
\hline $6-8$ cell & A \\
\hline
\end{tabular}

At the same time we were came in the IVF lab for checking of embryo status and cleavage. A series of cell division after fertilization was seen in which that net size of embryos would be remain same due to $S$ and $M$ cell cycle only involved, G1 \& G2 phase not involved in embryogenesis, but following DNA synthesis mitosis results in cells of approximately equal. In human there are three cleavage divisions from PN stage to 2 cells or 4 cells and 4 cells to 8 cells and so on.

\section{Embryo Transfer}

Embryo transfers (ET) was performed in 233 patients going for infertility treatment of IVF / ICSI procedures. 935 Embryos was selected from 2702 developing embryos for embryo transfer (ET) was performed in 233 patients by using a soft transfer catheter (Sydney IVF Embryo Transfer Set Cook, Ref \# K-JETS-7019-SIVF) and in some difficult ET we were used Gynetics Stellet ET Catheter (REF \# 4219 set) under transabdominal ultrasonographic guidance with filled urinary bladder.

Table 4 : Transfer of embryo on day-3 and its correlation with pregnancy

\begin{tabular}{|c|c|c|c|c|c|}
\hline \multirow{2}{*}{ Cell stage } & \multicolumn{4}{|c|}{ 'Grade } \\
\cline { 2 - 6 } & No. of Patients & Pregnant & $\begin{array}{c}\text { Non } \\
\text { pregnant }\end{array}$ & Pregnant & $\begin{array}{c}\text { Non } \\
\text { pregnant }\end{array}$ \\
\hline & 105 & 36 & 34 & 11 & 24 \\
\hline 8-cell & 128 & 48 & 25 & 17 & 38 \\
\hline Total Patients & 233 & 84 & 59 & 28 & 62 \\
\hline \multicolumn{2}{|c|}{ Percentage of conceptions } & $36.05 \%$ & $25.32 \%$ & $12.01 \%$ & $26.60 \%$ \\
\hline
\end{tabular}

\section{Results:}

Normal fertilization rate was defined as number of oocytes containing two pronuclei (2PN) divided by total number of mature oocyte (MII). Polyploid or polyspermy rate was defined as number of oocyte containing $>2 \mathrm{PN}$ divided by total no. of mature oocytes (MII). Embryo quality were defined as number of 4 - cell embryos on day 2 and number of 8 - cell embryos on day 3 . Overall embryo development in-vitro and conceiving rate was seen $48.06 \%$ after embryo transfer of both grades A and Grade B embryos in 233 patients in different age group with different endometrium thickness and multiple embryos transfer in one uterus depend on patient previous history. 
We are discussing and concluding morphological evaluation and selection of embryo is very important tool for in-vitro embryo development and embryos transfer in ART cycles. We were seen two pronuclei (2PN) center in the cytoplasm after conventional IVF / ICSI is good indicator of fertilization. That zygote was discarded who had shown 3PN/4 PN or multi nucleation. We are seen highest reproductive potential in equally blastomeres, no fragmentation, tightly bound eight celled blastomere on day three embryos. Those embryos showed early compaction have more potential to develop in blastocyst in-vitro as well as higher chance of implantation rate. We found higher embryo developed and early compaction in-vitro and higher implantation rate in Grade A embryos in comparison transferred of Grade B embryos.

\section{Time Scale:}

I have done morphological evaluation of human embryos from Day 1 to Day 3 was started from June 2017 to April 2020 on $>2702$ embryos or $\mathbf{2 3 3}$ patients going for the infertility treatment of IVF / ICSI procedures. 935 Embryos was selected for transfer and ET was performed in $\mathbf{2 3 3}$ patients. After this study we calculated achieving the conceiving rate was $48.06 \%$ based on morphology of embryo quality and grade.

\section{REFERENCES}

[1]. Fernando J. Prados, Sophie Debrock, Josephine G. Lemmen, and Inge Agerholm, Human Reproduction, Vol.27, No.S1 pp. i50-i71, 2012, doi:10.1093/humrep/des224.

[2]. Alikani M, Cohen J, Tomkin G, Garrisi GJ, Mack C, Scott RT. Human embryo fragmentation in vitro and its implications for pregnancy andimplantation. Fertil Steril 1999;71:836-842.

[3]. Asch, RH. Li, HP. Balmaceda, JP. Weckstein LN \& Stone, SC. (1991). Severe ovarian hyperstimulation syndrome in assisted reproductive technology: definition of high risk groups. Human Reproduction, Vol.6, No.10, pp. 1395-1399.

[4]. Depa-Martynow M, Jedrzejczak P, Pawelczyk L. Pronu $\neg$ clear scoring as a predictor of embryo quality in in vitro fertilization program. Folia Histochem Cytobiol. 2007; 45 Suppl 1: S85-89.

[5]. Hyung Jun Kim, Hye Jin Yoon et al. Evaluation of human embryo development in in vitro fertilization- and intracytoplasmic sperm injection-fertilized oocytes: A time-lapse study, https://doi.org/10.5653/cerm.2017.44.2.90 ,pISSN 2233-8233 · eISSN 2233-8241,Clin Exp Reprod Med 2017;44(2):90-95.

[6]. Alpha Scientist in Reproductive Medicine and ESHRE Special Interest Group of Embryology: The Istambul consensus workshop on embryoassessment: proceedings of an expert meeting. Hum Reprod 2011,26:1270-1283.

[7]. Hardarson T, Ahlström A, Rogberg L, Botros L, Hillensjö T, Westlander G,et al: Non-invasive metabolomic profiling of Day 2 and 5 embryo culture medium: a prospective randomized trial. Hum Reprod 2012, 27:89-96.

[8]. Uyar A, Seli E: Embryo assessment strategies and their validation for clinical use: a critical analysis of methodology. Curr Opin Obstet Gynecol 2012, 24:141-150.

[9]. Gianaroli L, Magli MC, Ferraretti AP, Fortini D, Greco N: Pronuclear morphology and chromosomal abnormalities as scoring criteria for embryo selection. Fertil Steril 2003, 80:341349.

[10]. Sara J. Denniset, Michael A. Thomas et al. Embryo morphology score on day 3 is predictive of implantation and live birth rates, 
Journal of Assisted Reproduction and Genetics, Vol. 23, No. 4, April 2006 (C _ 2006) DOI: 10.1007/s10815-006-9027-3

[11]. Shubhangi Kakade, Amit Kamat, Savita Datar, First mitotic division: criterion for selection of potential IVF embryo - A Systematic Literature Review, The International Journal Of Engineering And Science (IJES),Volume 4, Issue 4, Pages PP.41-48, 2015, ISSN (e): 2319 - 1813 ISSN (p): $2319-1805$.

[12]. Martin Greuner and Markus Montag, Morphological Selection of Gametes and Embryos: 2PN/Zygote.

[13]. Nicoli et al. Journal of Ovarian Research 2013, $6: 1$

http://www.ovarianresearch.com/content/6/1/1, Pronuclear morphology evaluation in in vitro fertilization (IVF) / intracytoplasmic sperm injection (ICSI) cycles: a retrospective clinical review.

[14]. Tomasz Bączkowski, Rafał Kurzawa1, Methods of embryo scoring in in vitro fertilization, Received: 3 November 2003; accepted: 12 January 2004. Vol. 4, No. 1, Reproductive Biology.

[15]. Franken DR, Claasens OE, Henkel RR. Sperm Preparation Techniques, X/Y Chromosome Separation. In: Acosta AA, Kruger TF (Eds). Human Spermatozoa in Assisted Reproduction.2nd edn. USA: Informa Healthcare 1996.p.277-94.

[16]. World Health Organization. (2010). WHO laboratory manual for the examination and processing of human semen. Fifth edition. 2010, Geneva

[17]. Revel A. Defective endometrial receptivity. 2012. Fertil Steril. 97 (5): 1028-32.

[18]. Rezazadeh Valojerdi M, Eftekhari-Yazdi P, Karimian L, Hassani F, Movaghar B. 2009. Vitrification versus slow freezing gives excellent survival, post warming embryo morpholo $\neg$ gy and pregnancy outcomes for human cleaved embryos. J Assist Reprod Genet. 26(6): 347-354.

[19]. Shapiro BS, Daneshmand ST, Garne FC, Aguirre M, Hudson C. 2014. Clinical rationale for cryopreservation of entire embryo cohorts in lieu of fresh transfer. Fertil Steril 102: 3-9.

[20]. Santos MA, Kuijk EW, Macklon NS. 2010. The impact of ovarian stimulation for IVF on the developing embryo. Reproduction. 139(1): 2334.

[21]. Montag, M., Liebenthron, J., Ko“ster, M., 2011. Which morphological scoring system is relevant in human embryo development? Placenta 32, S252-S256.

[22]. Song T, Liu L, Zhou F, Lin XN, Zhang SY. 2009. Frozen-thawed embryo transfer (FET) versus fresh embryo transfer in clinical pregnancy rate during in vitro fertilization-embryo transfer. Zhonghua Yi Xue Za Zhi. 89: 2928-2930.

[23]. Speroff L, Glass RH, Kase NG. 1999. Infertility. In: Clinical gynecologic endocrinology and infertility. 6th ed. Baltimore, USA: Lippincott Williams-Wilkins; p. 201-46.

[24]. Victoria Habibzadeh1 M.D., Sayed Noureddin Nematolahi Mahani2 Ph.D., Hadiss Kamyab1 M.D., The correlation of factors affecting the endometrial thickness with pregnancy outcome in the IUI cycles, Iranian Journal of Reproductive Medicine Vol.9. No.1. pp: 41-46, Winter 2011

[25]. Shoukir Y, Campana A, Farley T, Sakkas D. 1997. Early cleavage of in-vitro fertilized human embryos to the 2-cell stage: a novel indicator of embryo quality and viability. Hum Reprod. 12 (7): 1531-1536.

[26]. Scott L. Pronuclear scoring as a predictor of embryo development. 2003. Reprod Biomed Online. 6(2): 201-214.

[27]. Okuhue JE, Onuh SO, Ebeigbe P, Shaibu I, Wada I, et al. (2009) The effect of endometrial 
thickness on in vitro fertilization (IVF)-embryo transfer/ intracytoplasmic sperm injection (ICSI) outcome. Afr J Reprod Health 13: 113121.

[28]. Henkel RR, Schill W-B. 2003. Sperm preparation for ART. Reproductive Biology and Endocrinology.

\section{Cite this article as :}

Ram Dayal, Sumer Singh, Prabhat Kumar, Kamla Singh, Pawan Kumar Tripathi, Rajni Gupta, Sumit Singhal , "Morphological Evaluation and Grading of Human Embryo Quality from Day - 1 to Day - 3 Embryos for Optimum Conceiving Rate", International Journal of Scientific Research in Science and Technology (IJSRST), Online ISSN : 2395-602X, Print ISSN : 2395-6011, Volume 7 Issue 5, pp. 225-236, September-October 2020. Available at doi : https://doi.org/10.32628/IJSRST207546

Journal URL : http://ijsrst.com/IJSRST207546 